\title{
Overlapping gene structure of human VLCAD and DLG4
}

\author{
Changcheng Zhou, Bruce Blumberg* \\ Department of Developmental and Cell Biology, University of California, Irvine, 5205 McGaugh Hall, Irvine, CA 92697-2300, USA
}

Received 19 August 2002; received in revised form 16 December 2002; accepted 20 December 2002

Received by A. Sippel

\begin{abstract}
Very-long-chain acyl-CoA dehydrogenase (VLCAD) is a major enzyme catalysing the first step in mitochondrial $\beta$-oxidation of longchain fatty acids. During analysis of the $V L C A D$ promoter, we discovered that another gene, discs-large-related 4 (DLG4), overlaps $V L C A D$ and is transcribed in the opposite direction. DLG4 encodes postsynaptic density-95 (PSD95) protein, which plays critical roles in the formation and maintenance of synaptic junctions. The transcription start site of the VLCAD gene was determined by primer extension analysis and the overlapping structure of $V L C A D$ and $D L G 4$ was clarified. VLCAD and DLG4 are arranged in a head-to-head orientation on chromosome $17 \mathrm{p} 13$, and share a 245 bp overlapping region that contains part of $D L G 4$ exon 1 and the entire exon 1 of $V L C A D$ including 62 bp of protein coding sequence. Despite the overlap of their $5^{\prime}$ ends, $D L G 4$ and $V L C A D$ exhibit peak mRNA expression in different tissues, suggesting that they are independently regulated at the transcriptional level. Interestingly, $V L C A D$ and $D L G 4$ genes do not overlap in the mouse or Drosophila genomes.
\end{abstract}

(C) 2003 Elsevier Science B.V. All rights reserved.

Keywords: Overlapping gene; Quantitative real-time reverse transcription-polymerase chain reaction; Primer extension; Mitochondria $\beta$-oxidation

\section{Introduction}

Very-long-chain acyl-CoA dehydrogenase (VLCAD) is the major enzyme catalysing the first dehydrogenation step in the mitochondria $\beta$-oxidation of very long chain fatty acids(Strauss et al., 1995; Andresen et al., 1996). Four enzymes with different chain length specificities participate in the initial step in the mitochondria $\beta$-oxidation of straight-chain fatty acids. These are VLCAD, which requires straight-chain acyl-CoA substrates lengths of $\mathrm{C}_{14}-\mathrm{C}_{20}$; long-chain acyl-CoA dehydrogenase (LCAD); medium- chain acyl-CoA dehydrogenase (MCAD) and short-chain acyl-CoA dehydrogenase (SCAD). Four distinct dehydrogenase deficiencies have been described, with VLCAD deficiency being documented to cause hepatocellular dysfunction, severe lipid accumulation in organs, and

Abbreviations: VLCAD, very-long-chain acyl-CoA dehydrogenase; DLG4, discs-large-related 4; PSD95, postsynaptic density-95; GAPDH, glyceraldehyde 3-phosphate dehydrogenase; AP-1, activator protein 1; AP-2, activation protein 2 ; Sp1, Specificity protein $1 ; \mathrm{NF}-\kappa \mathrm{B}$, nuclear factor kappa-B; C/EBP, CCAAT/enhancer binding protein; kb, kilobase(s); bp, base pair(s); RT-PCR, reverse transcription-polymerase chain reaction; UTR, untranslated region.

* Corresponding author. Tel.: + 1-949-824-8573; fax: + 1-949-824-4709.

E-mail address: blumberg@uci.edu (B. Blumberg). cardiac disease, particularly hypertrophic cardiomyopathy (Aoyama et al., 1995).

Human VLCAD cDNA and genomic sequences were reported (Orii et al., 1995; Strauss et al., 1995; Andresen et al., 1996). The VLCAD gene is about $5.4 \mathrm{~kb}$ long, contains 20 exons that encode a 655 -amino-acid protein and is located on chromosome 17p13. VLCAD differs from the other three acyl-CoA dehydrogenases in that it is a homodimer of $70 \mathrm{kDa}$ subunits bound to the inner membrane whereas the others are soluble enzymes found in the matrix. Since patients with VLCAD deficiency were first reported (Aoyama et al., 1993; Bertrand et al., 1993), more than 80 VLCAD mutations have been identified. These include nucleotide substitution, small and gross deletions and insertions, and small insertions/deletions. There is a distinct relationship between the severity of VLCAD deficiency and the nature of the mutations. Severely affected patients have mutations that result in complete loss-of-function, whereas patients with milder phenotypes have mutations that result in residual enzyme activity (Andresen et al., 1999). Little is currently known about the regulation of $V L C A D$ transcription. In this report, we analysed the $5^{\prime}$ region of $V L C A D$ and discovered another gene, discs-large-related 4 (DLG4), overlapping VLCAD. $D L G 4$ encodes the postsynaptic density-95 (PSD95) protein 
that is one of four known human proteins belonging to the discs-large-related (DLG) subfamily of the member of membrane-associated guanylate kinase (MAGUK) proteins (Gomperts, 1996). PSD95 interacts with both $N$-methyl-Daspartate (NMDA) receptors and Shaker-type potassium channels and plays an important role in the formation and maintenance of synaptic junction (Stathakis et al., 1999). $D L G 4$ is positioned in a head-to-head orientation with the $V L C A D$, such that the $5^{\prime}$-untranslated region of $D L G 4$ overlaps with the $5^{\prime}$-UTR and a part of the protein-coding region of $V L C A D$. VLCAD and DLG4 are both widely expressed in adult tissues but each has peak expression in different tissues. Thus, despite their shared $5^{\prime}$ regions, DLG4 and $V L C A D$ are likely to be differentially regulated at the level of transcription. Consistent with this model, the $V L C A D$ and DLG4 homologs are not overlapping in the mouse and Drosophila genomes.

\section{Materials and methods}

\subsection{Primer extension analysis}

Primer extension analysis was performed using the $V L C A D$ specific oligonucleotide primer VL-66R $\left(5^{\prime}\right.$ TCCTGCACGCCCACGTCCT-3'), located 66 bp upstream

(A)

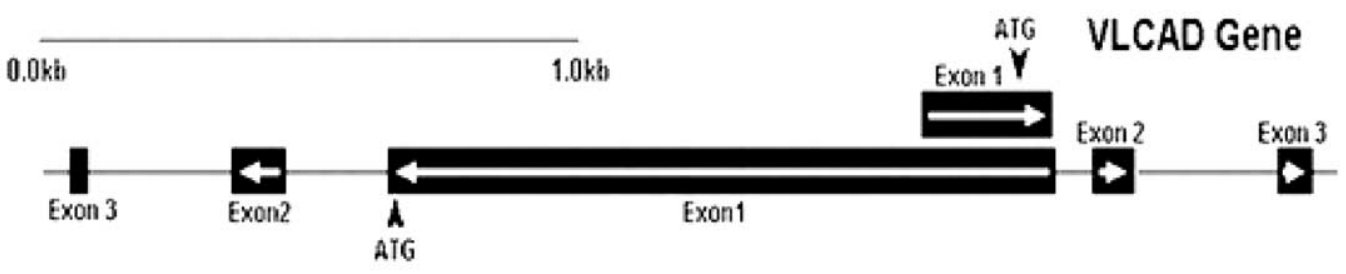

\section{DLG4 Gene}

(B)

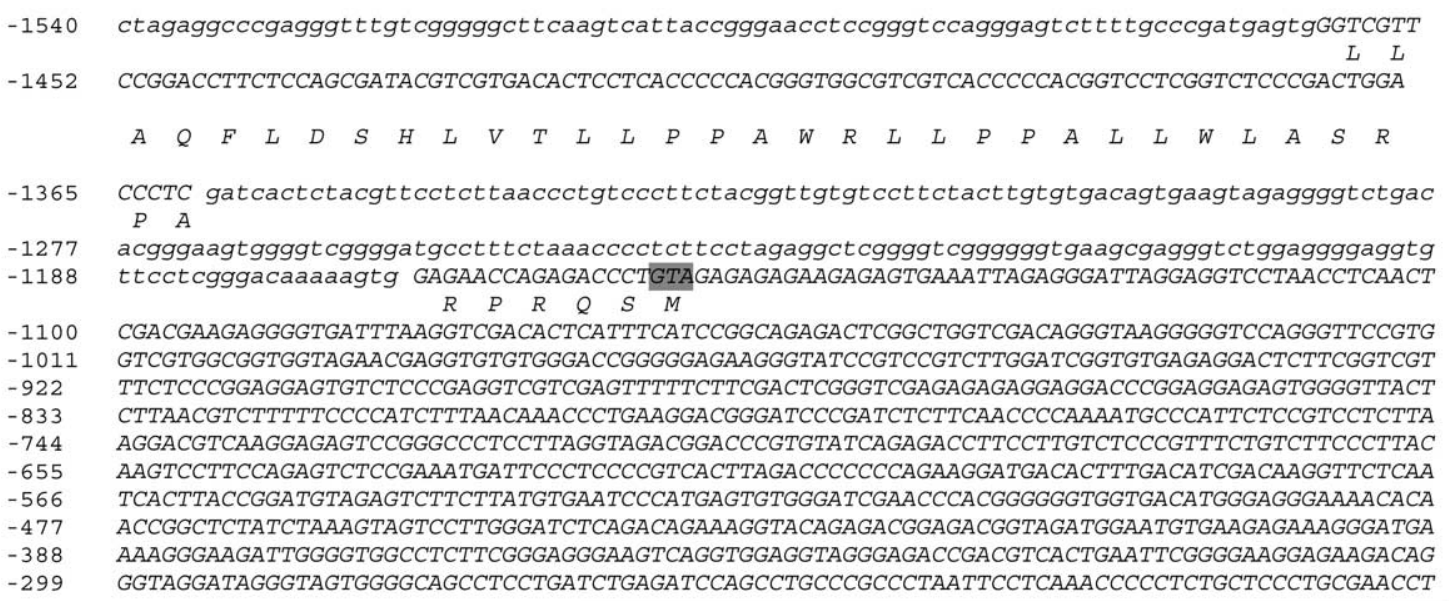

VLCAD 5' - GGCTCCAGGGAACTACAGCTCCCAGCATGCCCCGGGGCCCGCAACCGTCCGCCGCCCG -210 CTAGGGAGATTCAGTCGCCTTGCGTCCCGGC CCGAGGTCCCTTGATGTCGAGGGTCGTACGGGGCCCCGGGCGTTGGCAGGCGGCGGG

GTGCACTGTGGACGATGAGTCAGGGTTAGGGGCGCCAGGACGTGGGCGTGCAGGACGCGGGCGTGCAGGACGCCAGAGCTGGGTCAGAG - 121 CACGTGACACCTGCTACTCAGTCCCAATCCCCGCGGTCCTGCACCCGCACGTCCTGCGCCCGCACGTCCTGCGGTCTCGACCCAGTCTC CTCGAGCCAGCGGCGCCCGGAGAGATTCGGAGATGCAGGCGGCTCGGATGGCCGCGAGCTTGGGGCGGCAGCTGCTGAGGCTCGGGGGC GAGCTCGGTCGCCGCGGGCCTCTCTAAGCCTCTACGTCCGCCGAGCCTACCGGCGCTCGAACCCCGCCGTCGACGACTCCGAGCCCCCG $\begin{array}{lllllllllllllllllll}M & Q & A & A & R & M & A & A & S & L & G & R & Q & L & L & R & L & G & G\end{array}$

58 GGAAGgtct gtgtgtgacaagagggacggtgggcagcggecctgggcaccgggccggcactgaacccccactccCCacagCTCGCGG СCTTCCAGA - DLG4 5'

G S CTCACGGCGCTCCTGGGGCAGCCCCGGCCCGGCCCTGCCCGGCGGCCCTATGCCGGGGGTGCCGCTCAGgtaagtcaccgcagccttgg $\begin{array}{llllllllllllllllllllllllllll}L & T & A & L & L & G & Q & P & R & P & G & P & A & R & R & P & Y & A & G & G & A & A & Q\end{array}$

Fig. 1. (A) Schematic diagram of the genomic structure of human $V L C A D$ and $D L G 4$. The first three exons of $V L C A D$ and $D L G 4$ are shown in black boxes and arrows indicate the direction of transcription. Inferred ATG translation start codons are also indicated. (B) Nucleotide sequence of $1.7 \mathrm{~kb}$ of human genomic DNA comprising the first two exons of $V L C A D$ and $D L G 4$. The sequence is numbered beginning with the firstATG, which is the translation start of $V L C A D$. ATG translation start codons are highlighted in gray. Nucleotides in the overlapping region are boxed. The two exons of $V L C A D$ and $D L G 4$ are shown in uppercase and the amino acid sequence is shown below the exon nucleotides in one-letter code; introns are shown in lowercase. The oligonucleotide used for primer extension analysis is indicated by arrows positioned below. 
of the inferred $V L C A D$ ATG translation start codon. Total RNA was extracted from HeLa cells using the RNeasy kit (Qiagen) and primer extension performed using AMV reverse transcriptase (Primer Extension System; AMV Reverse Transcriptase Kit, Promega) according to the manufacturer's recommended protocols. Briefly, $5^{\prime}$ endlabeling reactions were conducted in a $10-\mu 1$ volume containing 10 pmol VL-66R, $1 \times$ T4 polynucleotide kinase buffer, $30 \mu \mathrm{Ci}\left[\gamma_{-}{ }^{32} \mathrm{P}\right] \mathrm{ATP}$ and 10 units $\mathrm{T}_{4}$ polynucleotide kinase. Primer extension reactions were conducted in $20 \mu \mathrm{l}$ volume containing $10 \mu \mathrm{g}$ total RNA, 1 pmol ${ }^{32} \mathrm{P}$-labeled primer, $2.8 \mathrm{mM}$ sodium pyrophosphate, $1 \times \mathrm{AMV}$ buffer and 2 units AMV reverse transcriptase. The transcription start site was mapped by electrophoresis on a $6 \%$ denaturing polyacrylamide gel and comparison with a DNA sequencing reaction from the $V L C A D$ promoter using the same primer.

\subsection{Quantitative real-time $R T-P C R$ analysis}

Human multiple tissue cDNA panels, which contain sets of first-strand cDNA, generated from different human tissues were commercially obtained (BD Clontech). Quantitative real time PCR was performed using the following primer sets: VLCAD (5'-GTCCTTTGCTGTGGGAATGT$\left.3^{\prime}\right) \quad\left(5^{\prime}\right.$-CAAGTGGTCTCCTCCACCAT-3 $\left.{ }^{\prime}\right)$, DLG4 $\left(5^{\prime}-\right.$ ACCAGATCCTGTCGGTCAAC-3') (5'-TGCTGTTCATGAGCTGTTCC-3'), or GAPDH (5'-GGCCTCCAAGGAGTAAGACC-3') (5'-AGGGGAGATTCAGTGTGGTG-3'), with the SYBR green PCR kit (Applied Biosystems) in a DNA Engine Opticon - Continuous Fluorescence Detection System (MJ Research). All samples were analysed in duplicate and quantitated by the comparative $\mathrm{Ct}$ method for relative quantitation of gene expression, normalized to GAPDH (Livak and Schmittgen, 2001).

\section{Results and discussion}

\subsection{Characterization of the $5^{\prime}$ region and transcription start site of VLCAD gene}

The VLCAD gene, located on chromosome $17 \mathrm{p} 13$, is about $5.4 \mathrm{~kb}$ long and consists of 20 exons and 19 introns (Orii et al., 1995). A BLAST search of the draft human genome using the VLCAD gene sequence (GenBank: SEG_D78279S) identified a chromosome 17-derived clone HRPC4G17 (GenBank: AC003688) that contains the entire $V L C A D$ gene on its plus strand. This $98-\mathrm{kb}$ PAC clone was sequenced by the Whitehead Institute/MIT center for Genome Research for the human chromosome 17 genome sequencing project. Further analysis of the upstream region of $V L C A D$ gene identified another previously described gene, DLG4 (Stathakis et al., 1997). The DLG4 gene encompasses approximate $30 \mathrm{~kb}$ and is composed of 22 exons. The HRPC4G17 clone contains the first 10 exons of DLG4 gene on its minus strand. Of particular interest is the fact that the $5^{\prime}$ end of $D L G 4$ gene overlaps with the $V L C A D$ gene and its transcription start site extends to the coding region of $V L C A D$ exon 1 (Fig. 1A).

The genomic structure and transcription start site of DLG4 were reported (Stathakis et al., 1999). However, the transcription start site of $V L C A D$ was unknown. We therefore conducted primer extension analysis of VLCAD. Total RNA from HeLa cells, which express the VLCAD mRNA (data not shown), was used to define the VLCAD transcription start site. Several groups have characterized the VLCAD cDNA. Only one major transcript was detected in a variety of human tissues and the mRNA size corresponds closely to the size of the VLCAD cDNA sequence (Aoyama et al., 1995; Orii et al., 1995; Strauss et al., 1995; Andresen et al., 1996). The primer used for primer extension analysis is located $66 \mathrm{bp}$ upstream of $V L C A D$ translation start site, within the $V L C A D$ exon 1 and transcription region. Primer extension revealed a single, distinct $113 \mathrm{bp}$ band (Fig. 2). This indicates that the major $V L C A D$ transcription start site is located 179 nucleotides upstream from the inferred ATG-translation initiation codon

\section{G A T C HeLa Marker}

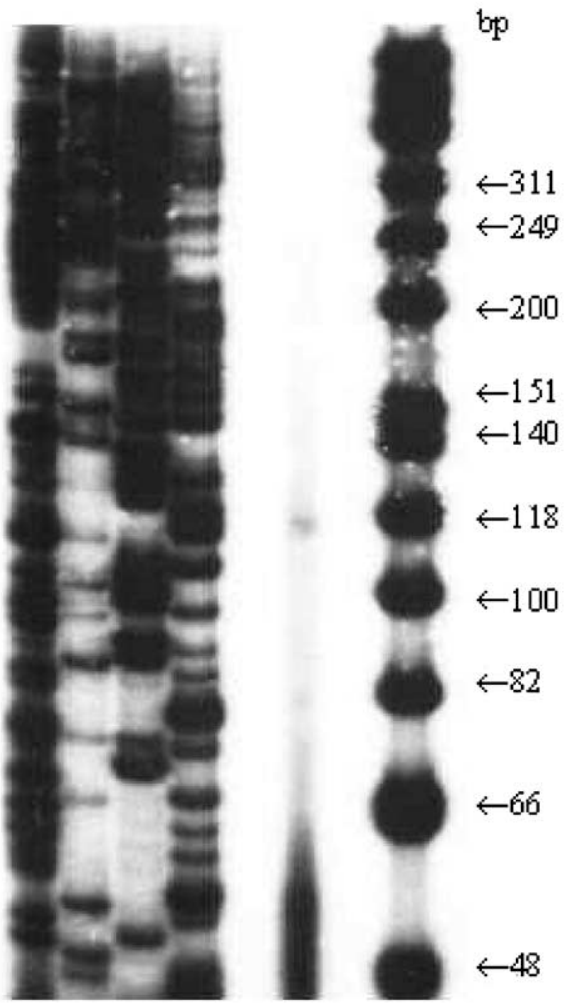

Fig. 2. Primer extension analysis of $V L C A D$. VLCAD specific primer VL66R was hybridized to $10 \mu \mathrm{g}$ of total RNA from HeLa cells and extended with reverse transcriptase. The first four lanes represent a DNA sequence ladder generated by dideoxy chain termination reactions using VL-66R as primer and $\operatorname{ddGTP}(\mathrm{G})$, ddATP(A), $\operatorname{ddTTP}(\mathrm{T})$ or $\operatorname{ddCTP}(\mathrm{C})$. The fifth lane is the primer extension product. A $113 \mathrm{bp}$ band represents the major primer extension product of $V L C A D$ gene. The last lane is a DNA size marker (lane Marker). 
of $V L C A D$. This position is within DLG4 exon 1, 245 nucleotides downstream from its transcription start site (Fig. 1B).

After clarifying the transcription start site of the $V L C A D$ gene, we found that $V L C A D$ and DLG4 share 245 nucleotides at their $5^{\prime}$ ends, and are transcribed in opposite directions. The sequence of the overlapping region of $V L C A D$ and $D L G 4$ genes was confirmed by sequencing of a $1 \mathrm{~kb}$ genomic PCR fragment obtained directly from human genomic DNA using a primer set that corresponds to both $V L C A D$ and $D L G 4$ (data not shown).

The overlapping gene structure of $V L C A D$ and $D L G 4$ is unusual in the human genome. The N-terminus of the $D L G 4$ gene product, the PSD95 protein, is modified by thioesterlinked palmitate, a 16-carbon saturated fatty acid that targets PSD95 to cell membranes (Topinka and Bredt, 1998). PSD95 is a major palmitoylated protein in intact cells and palmitoylation is a critical regulatory mechanism for receptor interactions with PSD95. On the other hand, VLCAD is the major enzyme for the degradation of palmitate during its first step of $\beta$-oxidation in vivo. Therefore, the unusual structure of these two genes suggests a possible link between the use of palmitate as an energy substrate and the signal transduction pathway mediated by PSD95.

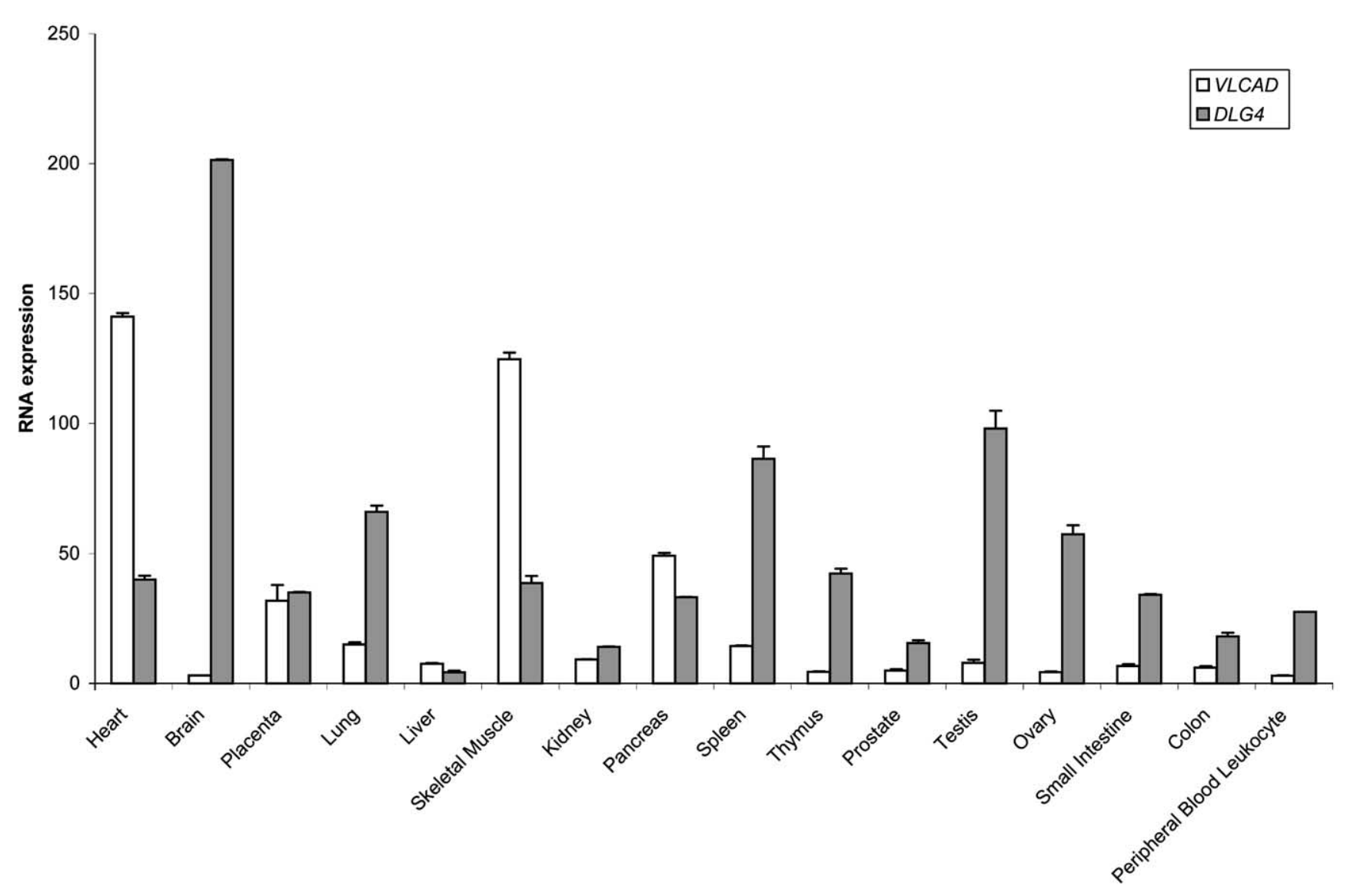

Fig. 3. Tissue-specific expression of $V L C A D$ and $D L G 4$. Real time RT-PCR was used to determine which human adult tissues expressed $V L C A D$ and $D L G 4$.

Data are shown in the form of RNA expression levels normalized to GAPDH controls. Values represent the average of duplicates \pm range.
Human multiple tissue cDNA panels, which contain sets of first-strand cDNA generated from different human tissues were used to investigate the tissue specific distribution of $V L C A D$ and $D L G 4$ by quantitative real-time RT-PCR. Although the overlapping region of $V L C A D$ and $D L G 4$ covers the entire $V L C A D$ exon 1 including 62 bp of coding region, transcription of these two genes is not impaired by their overlap. Both genes can be co-expressed in the same tissues, but exhibit different patterns of maximal expression (Fig. 3). DLG4 mRNA is most abundant in brain with prominent expression detected in heart, placenta, lung, pancreas, spleen, thymus, testis, ovary and small intestine. In contrast, $V L C A D$ is highly expressed in heart and skeletal muscle with lower levels detectable in placenta and pancreas. Both genes were expressed at lower, but detectable levels in the other tissues examined. We infer from the expression of their mRNAs that these two genes share common regulatory elements but also have distinct tissue-specific elements required for high-level expression.

Computer analysis of the upstream regions of $V L C A D$ and $D L G 4$ genes including their overlapping region was performed using promoter scan II (http://biosci.cbs.umn. edu/software/software.html) and MatInspector 2.2 (http:// 
transfac.gbf.de/TRANSFAC). This analysis identified two potential TATA-less promoters and potential binding sites for several transcription factors including Sp1, AP-1, AP-2, $\mathrm{C} / \mathrm{EBP}$ and NF-кB. The computer prediction of $V L C A D$ gene's promoter region and AP-2 binding site are consistent with a published report (Zhou et al., 1999). Further studies to characterize transcription factors essential for the correct regulation of both these promoters will provide insight into the mechanism governing the regulation of both genes at the transcriptional level.

\subsection{Evolutionary analysis of VLCAD and DLG4 gene loci}

Overlapping genes occur frequently in prokaryotic, viral and mitochondrial genomes but only rarely in most eukaryotic genomes, including the human genome (Shintani et al., 1999). Since the first two overlapping genes reported by Emi et al. (Emi et al., 1988), a small number of human overlapping genes have been identified (Ubeda et al., 1999; Faurholm et al., 2001; Dan et al., 2002). Although a few cases have been reported, anti-parallel overlapping of exons of functional protein coding genes is quite rare (Nicolaides et al., 1995; Herzog et al., 1997; Cooper et al., 1998).

To investigate the evolution of this overlapping organization, we examined the structure of VLCAD and DLG4 in the draft Drosophila and mouse genomes. The Drosophilagene CG7461 (FlyBase ID: FBgn0034432) that encodes VLCAD is located on chromosome 2R 56C9-56C10. Discs large 1 (FlyBase ID: FBgn0001624), the fly ortholog of the human $D L G$ family is located on the $\mathrm{X}$ chromosome at locus 10B11-10B12. A BLAST search of the mouse genome with VLCAD (XM_126408) and DLG4 (NM_007864) revealed that these genes are both located on chromosome 6 at 11B3-B4 in a head-to-head orientation. In contrast to the human genes, the mouse genes do not overlap and contain a gap of $3.48 \mathrm{~kb}$ between them. This suggests that the $V L C A D$ and $D L G 4$ genes were originally separated but have become co-localized during the course of evolution. Further analysis of the genomic structures of these genes in other organisms (as the genomic sequences become available) will allow one to deduce when this colocalization event occurred during the course of vertebrate evolution.

\subsection{Conclusion}

We identified two overlapping genes $V L C A D$ and $D L G 4$ in the human genome. The transcription start site of $V L C A D$ was determined and it was shown that VLCAD and DLG4 are organized in a head-to-head orientation sharing a $245 \mathrm{bp}$ overlapping region that encompasses part of $D L G 4$ exon 1 and the entire first exon of $V L C A D$ including $62 \mathrm{bp}$ of protein-coding region. Both genes were expressed in all adult tissues examined although sites of peak expression were different. VLCAD and DLG4 exonic sequences do not overlap in the Drosophila or mouse genomes, suggesting that the loss of intergenic sequences happened late in mammalian evolution.

\section{References}

Andresen, B.S., Bross, P., Vianey-Saban, C., Divry, P., Zabot, M.T., Roe, C.R., Nada, M.A., Byskov, A., Kruse, T.A., Neve, S., Kristiansen, K., Knudsen, I., Corydon, M.J., Gregersen, N., 1996. Cloning and characterization of human very-long-chain acyl-CoA dehydrogenase cDNA, chromosomal assignment of the gene and identification in four patients of nine different mutations within the VLCAD gene. Hum. Mol. Genet. 5, 461-472.

Andresen, B.S., Olpin, S., Poorthuis, B.J., Scholte, H.R., Vianey-Saban, C., Wanders, R., Ijlst, L., Morris, A., Pourfarzam, M., Bartlett, K., Baumgartner, E.R., deKlerk, J.B., Schroeder, L.D., Corydon, T.J., Lund, H., Winter, V., Bross, P., Bolund, L., Gregersen, N., 1999. Clear correlation of genotype with disease phenotype in very-long-chain acylCoA dehydrogenase deficiency. Am. J. Hum. Genet. 64, 479-494.

Aoyama, T., Uchida, Y., Kelley, R.I., Marble, M., Hofman, K., Tonsgard, J.H., Rhead, W.J., Hashimoto, T., 1993. A novel disease with deficiency of mitochondrial very-long-chain acyl-CoA dehydrogenase. Biochem. Biophys. Res. Commun. 191, 1369-1372.

Aoyama, T., Souri, M., Ushikubo, S., Kamijo, T., Yamaguchi, S., Kelley, R.I., Rhead, W.J., Uetake, K., Tanaka, K., Hashimoto, T., 1995. Purification of human very-long-chain acyl-coenzyme A dehydrogenase and characterization of its deficiency in seven patients. J. Clin. Invest. 95, 2465-2473.

Bertrand, C., Largilliere, C., Zabot, M.T., Mathieu, M., Vianey-Saban, C., 1993. Very long chain acyl-CoA dehydrogenase deficiency: identification of a new inborn error of mitochondrial fatty acid oxidation in fibroblasts. Biochim. Biophys. Acta 1180, 327-329.

Cooper, P.R., Smilinich, N.J., Day, C.D., Nowak, N.J., Reid, L.H., Pearsall, R.S., Reece, M., Prawitt, D., Landers, J., Housman, D.E., Winterpacht, A., Zabel, B.U., Pelletier, J., Weissman, B.E., Shows, T.B., Higgins, M.J., 1998. Divergently transcribed overlapping genes expressed in liver and kidney and located in the $11 \mathrm{p} 15.5$ imprinted domain. Genomics 49, 38-51.

Dan, I., Watanabe, N.M., Kajikawa, E., Ishida, T., Pandey, A., Kusumi, A., 2002. Overlapping of MINK and CHRNE gene loci in the course of mammalian evolution. Nucleic Acids Res. 30, 2906-2910.

Emi, M., Horii, A., Tomita, N., Nishide, T., Ogawa, M., Mori, T., Matsubara, K., 1988. Overlapping two genes in human DNA: a salivary amylase gene overlaps with a gamma-actin pseudogene that carries an integrated human endogenous retroviral DNA. Gene 62, 229-235.

Faurholm, B., Millar, R.P., Katz, A.A., 2001. The genes encoding the type II gonadotropin-releasing hormone receptor and the ribonucleoprotein RBM8A in humans overlap in two genomic loci. Genomics 78, 15-18.

Gomperts, S.N., 1996. Clustering membrane proteins: It's all coming together with the PSD-95/SAP90 protein family. Cell 84, 659-662.

Herzog, H., Darby, K., Ball, H., Hort, Y., Beck-Sickinger, A., Shine, J., 1997. Overlapping gene structure of the human neuropeptide $Y$ receptor subtypes Y1 and Y5 suggests coordinate transcriptional regulation. Genomics 41, 315-319.

Livak, K.J., Schmittgen, T.D., 2001. Analysis of relative gene expression data using real-time quantitative PCR and the 2(-Delta Delta C(T)) method. Methods 25, 402-408.

Nicolaides, N.C., Kinzler, K.W., Vogelstein, B., 1995. Analysis of the $5^{\prime}$ region of PMS2 reveals heterogeneous transcripts and a novel overlapping gene. Genomics 29, 329-334.

Orii, K.O., Aoyama, T., Souri, M., Orii, K.E., Kondo, N., Orii, T., Hashimoto, T., 1995. Genomic DNA organization of human mitochondrial very-long-chain acyl-CoA dehydrogenase and mutation analysis. Biochem. Biophys. Res. Commun. 217, 987-992.

Shintani, S., O’Huigin, C., Toyosawa, S., Michalova, V., Klein, J., 1999. 
Origin of gene overlap: the case of TCP1 and ACAT2. Genetics 152, $743-754$.

Stathakis, D.G., Hoover, K.B., You, Z., Bryant, P.J., 1997. Human postsynaptic density-95 (PSD95): location of the gene (DLG4) and possible function in nonneural as well as in neural tissues. Genomics 44 , $71-82$.

Stathakis, D.G., Udar, N., Sandgren, O., Andreasson, S., Bryant, P.J., Small, K., Forsman-Semb, K., 1999. Genomic organization of human DLG4, the gene encoding postsynaptic density 95 . J. Neurochem. 73, 2250-2265.

Strauss, A.W., Powell, C.K., Hale, D.E., Anderson, M.M., Ahuja, A., Brackett, J.C., Sims, H.F., 1995. Molecular basis of human mitochondrial very-long-chain acyl-CoA dehydrogenase deficiency causing cardiomyopathy and sudden death in childhood. Proc. Natl. Acad. Sci. USA 92, 10496-10500.

Topinka, J.R., Bredt, D.S., 1998. N-terminal palmitoylation of PSD-95 regulates association with cell membranes and interaction with $\mathrm{K}^{+}$ channel Kv1.4. Neuron 20, 125-134.

Ubeda, M., Schmitt-Ney, M., Ferrer, J., Habener, J.F., 1999. CHOP/ GADD153 and methionyl-tRNA synthetase (MetRS) genes overlap in a conserved region that controls mRNA stability. Biochem. Biophys. Res. Commun. 262, 31-38.

Zhou, Y., Kelly, D.P., Strauss, A.W., Sims, H., Zhang, Z., 1999. Characterization of the human very-long-chain acyl-CoA dehydrogenase gene promoter region: a role for activator protein 2. Mol. Genet. Metab. 68, 481-487. 